\title{
PROGRESSO SCIENTIFICO E INNOVAZIONE TECNOLOGICA: ASPETTI ETICI E GIURIDICI
}

\author{
SALVATORE VECA $(*)$
}

\begin{abstract}
SuNTO. - Il mio intervento mette a fuoco alcuni temi principali: l'immagine sociale della scienza, gli effetti dell'innovazione tecnologica e la nozione di qualità di vita. Propongo sette dilemmi generati dall'analisi dei temi principali e cerco di chiarire $i$ ) in che senso sia urgente un nuovo contratto sociale fra scienza e società; ii) perché abbiamo bisogno di una cultura matura e pluralistica dell'innovazione; iii) perché dobbiamo adottare una nozione multidimensionale di qualità della vita.
\end{abstract}

$* * *$

ABSTRACT. - My paper focuses on several main themes: the social image of science, the effects of technological innovation and the notion of the quality of life. I propose seven dilemmas generated by the analysis of these main themes, and try to clarify $i$ ) in what way a new social contract between science and society is urgent; ii) why we need a mature, pluralistic culture of innovation; iii) why we should adopt a multidimensional notion of the quality of life.

Che gli esiti della crescita della conoscenza scientifica e dell'innovazione tecnologica siano fra i più rilevanti fattori di miglioramento della qualità di vita di persone è un fatto per lo più incontrovertibile nella cultura contemporanea. Il riconoscimento di questo fatto assume spesso il carattere di una convinzione e di una credenza ordinaria. Tuttavia, non appena si mettano a fuoco la natura e le implicazioni della convinzione e della credenza ordinaria, si incappa immediatamente in una ampia gamma di dilemmi.

Alcuni dilemmi concernono la ricerca scientifica, altri sono generati dall'innovazione tecnologica, altri - infine - chiamano in causa la

(*) Istituto Lombardo Accademia di Scienze e Lettere, Milano, IUSS Pavia, Italia. E-mail: salvatore.veca@iusspavia.it 
stessa nozione di qualità di vita. E di qui, sono a loro volta chiamati in causa i criteri di valutazione etica della stessa, in un quadro culturale caratterizzato dal pluralismo dei valori che ci è contemporaneo. Basta pensare alla differenza fra etica o, meglio, etiche religiose, etiche laiche, prospettive consequenzialistiche, telelologiche e deontologiche, teorie dell'utilità sociale e teorie dei diritti, etiche delle virtù ed etiche comunitarie. Naturalmente, la distinzione fra i tre tipi di questioni è solo un artificio espositivo, dato che molto spesso essa è debole e porosa. E hanno luogo molte intersezioni fra i tre tipi individuati, per convenzione, come distinti. Ecco ora i sette dilemmi che propongo alla vostra riflessione.

1. Il primo dilemma riguarda la tensione e la variabile prossimità o distanza fra l'immagine scientifica e l'immagine manifesta della realtà e del mondo (e di noi nel mondo) nel senso di Wilfrid Sellars. I costi cognitivi di accesso agli sviluppi della conoscenza scientifica possono essere molto alti per ampie frazioni di popolazione. In molti casi, la scarsa o nulla padronanza concettuale dei linguaggi scientifici viene sostituita dalla fiducia che si basa sulla convinzione e sulla credenza ordinaria del fatto incontrovertibile, cui ci siamo prima riferiti.

Qui si formula il primo problema: come ridurre la distanza fra immagine scientifica e immagine manifesta della realtà e del mondo (e di noi nel mondo)? (Si osservi, a contrario, che si possono dare casi di revoca e ritiro della fiducia e di veri e propri processi di delegittimazione dei linguaggi "esperti".) Il primo problema è, in definitiva, un problema di buman divide epistemico, uno dei molti volti della disuguaglianza. La riduzione di questa disuguaglianza è dettata dai principi di una giustizia come equità epistemica di cittadinanza. E se si condividono i principi, si aprono la discussione, la ricerca e la valutazione dei metodi alternativi per ridurre la disuguaglianza epistemica. E questa è una prima tessera importante del mosaico di un'etica pubblica.

2. Un secondo dilemma, che concerne la cerchia dell'impresa scientifica, ha carattere sociale. Nelle nostre società assistiamo a una marcata revoca di fiducia nei confronti delle istituzioni e delle élites, siano esse politiche, economiche, epistemiche o culturali. La credenza ordinaria e la convinzione nel fatto incontrovertibile del nesso fra scienza e qualità di vita non sono immunizzate rispetto al rischio del ritiro di fiducia, cui ho prima accennato. Questo problema può essere formulato come quello della possibilità di una nuova alleanza o di 
nuovo contratto sociale fra la cerchia della ricerca e quella della cittadinanza. $\mathrm{O}$ un rinnovato legame di amicizia civica fra scienza e società.

Ciò ha a che vedere con il legame sociale, da un lato, e con la responsabilità del rendere conto e del mantenere le promesse a proposito del nesso scienza-qualità di vita da parte della comunità scientifica. Se si accetta questa formulazione del problema, si apre una discussione ragionevole sui metodi e sugli impegni per la sua soluzione più efficace. E questa è una seconda tessera importante del mosaico di un'etica pubblica.

3. Il terzo dilemma ha di nuovo carattere sociale. Conosciamo la gamma delle disuguaglianze che contraddistinguono i nostri modi di convivere: disuguaglianze di reddito, ricchezza, capitale sociale, capitale relazionale e culturale. Ci sfugge spesso una dimensione della disuguaglianza che possiamo definire disuguaglianza generazionale. Questa dimensione è trasversale a tutte le altre e chiama in causa la necessità che la nuova alleanza fra scienza e società sia costruita e rivolta in primo luogo ai giovani e, in particolare, ai bambini e alle bambine. Children first.

Le bambine e i bambini possono essere i più efficaci militanti nella causa della nuova alleanza fra scienza e società e, soprattutto, sono i cittadini e le cittadine future. Qui siamo al cuore e alla radice di una responsabilità civile nell'educare persone, ai tempi della loro fioritura, a conoscere e padroneggiare i fondamentali del nesso scienza-società. Ecco la terza tessera importante del mosaico di un'etica pubblica.

4. Consideriamo ora una famiglia di dilemmi generata dall'innovazione tecnologica. L'innovazione ha molti volti. Contraddistinta da un alto grado di improbabilità, essa irrompe nelle circostanze ordinarie, alterando il paesaggio abituale dei nostri modi di vivere e convivere. Essa genera incertezza in coloro su cui ha effetti o può avere effetti. L'incertezza investe non solo le nostre credenze epistemiche ordinarie (come nel caso degli sviluppi dell'impresa scientifica), ma anche direttamente i nostri criteri personali di valutazione etica. (L'impresa scientifica ci dice come stanno le cose. L'innovazione tecnologica e le sue applicazioni ci suggeriscono come fare cose, sapendo come esse stanno.) Quando i poteri causali, i poteri prima non disponibili di fare cose e di avere effetti prima non accessibili, si estendono e dilatano i confini del possibile, corrispondentemente si ampliano le nostre responsabilità morali e, quindi, giuridiche. Si generano aspet- 
tative che possono configurarsi come diritti delle persone, cui sono correlati doveri. L'innovazione tecnologica sembra chiedere, nell'incertezza, una cultura dell'innovazione stessa. Perché noi siamo sempre blandamente o severamente antiquati rispetto a noi stessi. E i nostri giudizi di valore sono letteralmente pregiudizi morali, dato che essi si sono consolidati su uno sfondo di credenze ormai più o meno lontano nel tempo.

Basti pensare, in proposito, ai dilemmi morali e giuridici generati, in virtù di applicazioni sofisticate dell'intelligenza artificiale, dalle macchine autonome, in cui nuove domande si formulano quanto alla responsabilità e ai diritti fondamentali delle persone in differenti prospettive etiche. La famiglia di effetti dell'innovazione genera quindi la domanda di una cultura matura e responsabile dell'innovazione e dei suoi molti volti, che pervenga a un punto di equilibrio riflessivo fra i criteri di valutazione e orientamento, vecchi e nuovi. Come dire: No Innovation without Representation (and Participation).

5. Se riflettiamo sugli elementi caratterizzanti di una cultura matura e responsabile dell'innovazione, possiamo incontrare una difficoltà. Il dilemma dipende qui dal fatto che tendono a prevalere, nella cultura diffusa nelle nostre società, un orientamento e un atteggiamento che, consapevolmente o meno, sottostimano l'importanza del nostro senso del passato. Sembra che un'immagine riduttiva dell'innovazione contragga i nostri orizzonti temporali sia per quanto riguarda il passato sia per quanto riguarda il futuro. Ci muoviamo come surfando sull'onda di un eterno presente. Una cultura dell'innovazione che azzera il senso del passato è una cultura povera e inadeguata.

Il passato è semplicemente un altro presente e possiamo interpretarlo come un repertorio di possibilità e di alternative. La conoscenza dei modi in cui le nostre controparti passate hanno pensato, valutato, lodato, negato, santificato o demonizzato le innovazioni del loro presente costituisce un ingrediente fondamentale per una cultura non riduzionistica e plurale dell'innovazione e ci orientano verso un futuro che può essere più degno di lode.

6. Una distinta classe di dilemmi concerne direttamente la nozione di qualità di vita di persone. Anche la qualità di vita è caratterizzata da una pluralità di dimensioni, cui deve essere rispondente una pluralità di parametri per la sua valutazione. La qualità di vita ha certamente una dimensione oggettiva e impersonale o intersoggettiva, ma ha anche una dimensione soggettiva e personale. L'essere una vita 
migliore o peggiore per chi la vive dipende dalla ponderazione appropriata dei diversi parametri. Seguendo l'approccio delle capacità di Amartya K. Sen, possiamo individuare un livello oggettivo di funzionamenti della persona e un livello soggettivo di capacità della persona di scegliere tra funzionamenti alternativi. E le ragioni della scelta sono coerenti con ciò che si prova a vivere quella particolare vita. Le persone aspirano a che le loro vite abbiano senso e significato in una rete di connessioni e relazioni. E questo, in fondo, è la caratteristica saliente del loro essere persone. Molto spesso, nella valutazione della qualità di vita, questa dimensione - che ha a che vedere con ciò che si prova a vivere le proprie vite - è trascurata a favore di una prospettiva unidimensionale o monistica per la metrica della qualità di vita. In particolare, in presenza delle circostanze del mutamento e dell'innovazione direttamente o indirettamente ascrivibili alla crescita della conoscenza scientifica, una visione monistica, e non pluralistica, dei molti volti della qualità di vita è miope e riduzionistica. Si consideri, inoltre, che nelle nostre società l'allungamento dell'aspettativa di vita delle persone rende particolarmente importante una piena rispondenza, nella valutazione, ai molti volti della qualità di vita di persone nel tempo.

Il problema che qui si formula ha a che vedere con lo sviluppo e la sofisticazione di un approccio costituivamente pluralistico alla qualità di vita di persone. Nella teoria, nelle istituzioni e nelle pratiche sociali, a partire dalle pratiche di cura in presenza di profondi cambiamenti cognitivi e applicativi nell'ambito delle scienze della vita e della biomedicina. Per questo, del resto, si parla ormai di Medical Humanities.

7. L'ultimo dilemma si genera, se immergiamo le nostre considerazioni sul nesso fra ricerca scientifica, innovazione tecnologica, etica e criteri di valutazione della qualità di vita di persone nel più vasto spazio di quanto vorremmo poter chiamare "umanità". Se adottiamo la prospettiva degli "occhi del resto dell'umanità", come sostiene il filosofo morale Adam Smith o la prospettiva dei cittadini del mondo dell'illuminista Voltaire, o quella del "pensiero largo" di Kant, l'illuminista del "sapere aude" e di Per la pace perpetua, si formula immediatamente la questione difficile del nesso fra ricerca e società, quando lo proiettiamo su scala globale. E, non dimenticando la leggendaria risposta di Albert Einstein a proposito dell'unica "razza umana", 
siamo indotti a interrogarci sulle responsabilità della comunità scientifica e delle comunità epistemiche, qui e ora, nei confronti di miliardi di coinquilini del pianeta. L'unico pianeta che, dopo tutto, condividiamo, come ci ha ricordato Stephen Hacking. Ci chiediamo allora: quali effetti può o deve avere il nesso scienza-società sui modi equi della condivisione dell'unico pianeta, taking seriously Humanity? E viene fatto di pensare che quest'ultima è una genuina questione di equità o giustizia globale che può o, forse, deve orientare la direzione delle nostre ricerche e motivare il confronto responsabile delle idee. 